\title{
Signal representation on the angular Poincaré sphere, based on second-order moments
}

\author{
Martin J. Bastiaans ${ }^{1, *}$ and Tatiana Alieva ${ }^{2}$ \\ ${ }^{1}$ Eindhoven University of Technology, Department of Electrical Engineering, P.O. Box 513, \\ 5600 MB Eindhoven, Netherlands \\ ${ }^{2}$ Universidad Complutense de Madrid, Facultad de Ciencias Físicas, Ciudad Universitaria s/n, Madrid 28040, Spain \\ *Corresponding author: m.j.bastiaans@tue.nl
}

Received December 17, 2009; accepted February 15, 2010;

posted February 19, 2010 (Doc. ID 121691); published March 31, 2010

\begin{abstract}
Based on the analysis of second-order moments, a generalized canonical representation of a two-dimensional optical signal is proposed, which is associated with the angular Poincaré sphere. Vortex-free (or zero-twist) optical beams arise on the equator of this sphere, while beams with a maximum vorticity (or maximum twist) are located at the poles. An easy way is shown how the latitude on the sphere, which is a measure for the degree of vorticity, can be derived from the second-order moments. The latitude is invariant when the beam propagates through a first-order optical system between conjugate planes. To change the vorticity of a beam, a system that does not operate between conjugate planes is needed, with the gyrator as the prime representative of such a system. A direct way is derived to find an optical system (consisting of a lens, a magnifier, a rotator, and a gyrator) that transforms a beam with an arbitrary moment matrix into its canonical form. (C) 2010 Optical Society of America
\end{abstract}

OCIS codes: $080.5084,080.2468,070.2590,080.2730,140.3295,080.4865$.

\section{INTRODUCTION}

It is common to use the angular Poincaré sphere for the representation of the polarization [1] (or, in other words, the spin components of the angular momentum) of a radiation beam. This approach has recently been generalized [2-6] to the case of Gaussian-type modes, which may possess orbital angular momentum. In the present paper we propose to use this formalism for the description of an arbitrary scalar two-dimensional signal, which might be deterministic or stochastic (i.e., completely coherent or partially coherent).

An optical beam is usually characterized by the ten central second-order moments of its Wigner distribution $[7,8]$, arranged in a $4 \times 4$ matrix $\mathbf{M}$. These moments provide information about the beam's transversal width, its far-field divergence, the $z$-component of the orbital angular momentum, its principal axes, several quality parameters, etc. [9]. Furthermore, based on the knowledge of the second-order moments, we can separate all beams into two groups: intrinsically isotropic beams on the one hand and anisotropic ones on the other [10]. To characterize the anisotropy, we use the Poincaré sphere.

After transforming an arbitrary signal into its canonical form, corresponding to a diagonal moment matrix $[10,11]$, we associate the resulting state with the intersection of the main meridian and the equator on the Poincaré sphere. However, the actual state of the original signal itself may be different from the one of its canonical form, which-as a matter of fact-always corresponds to zero vorticity.

In this paper we introduce a generalized canonical form that allows us to represent the signal as a point on the
Poincaré sphere. The latitude of this point corresponds to the vorticity of the beam (i.e., the value of the $z$-component of the orbital angular momentum), while its longitude corresponds to the orientation of the beam's principal axis. We show a way to express the latitude on the sphere directly in terms of the second-order moments. Moreover, we observe that the latitude is invariant when the beam propagates through a first-order optical system between conjugate planes. If we identify the vorticity of a beam with this latitude, we conclude that the vorticity can only change when the beam propagates through a non-conjugate system, with the gyrator as the prime representative.

We also show a direct way to find an optical system (in general consisting of a lens, a magnifier, a rotator, and a gyrator) that transforms a beam with an arbitrary moment matrix into its canonical form. Such a system has a clear physical meaning: (i) the lens cancels the quadratic phase front of the initial beam; (ii) the subsequent magnifier matches the beam's space moments with its spatialfrequency moments; (iii) the rotator aligns the beam to its principal axis; and (iv) the gyrator expresses the vorticity of the beam. The proposed approach may be useful for different applications such as beam characterization and signal analysis and synthesis.

\section{WIGNER DISTRIBUTION AND ITS TEN SECOND-ORDER MOMENTS}

We recall that the Wigner distribution $W(\mathbf{r}, \mathbf{q})$ of an optical signal with cross-spectral density function $\Gamma\left(\mathbf{r}_{1}, \mathbf{r}_{2}\right)$, is defined as [7] 


$$
W(\mathbf{r}, \mathbf{q})=\int \Gamma\left(\mathbf{r}+\frac{1}{2} \mathbf{r}^{\prime}, \mathbf{r}-\frac{1}{2} \mathbf{r}^{\prime}\right) \exp \left(-i 2 \pi \mathbf{q}^{t} \mathbf{r}^{\prime}\right) \mathrm{d} \mathbf{r}^{\prime}
$$

In the above expressions, $\mathbf{r}=[x, y]^{t}$ represents the transversal space coordinates $x$ and $y$ in the form of a column vector, and $\mathbf{q}=[u, v]^{t}$ is a similar representation of the spatial-frequency variables $u$ and $v$. For convenience, we use dimensionless space and spatial-frequency variables $\mathbf{r}$ and $\mathbf{q}$, normalized in the $x$ and $y$ directions to $w_{x, y}$ and $w_{x, y}^{-1}$, respectively. The definition (1) is valid for a general optical signal, which may be partially coherent; we recall that for a coherent signal $f(\mathbf{r})$, the cross-spectral density $\Gamma\left(\mathbf{r}_{1}, \mathbf{r}_{2}\right)$ reduces to the product $f\left(\mathbf{r}_{1}\right) f^{*}\left(\mathbf{r}_{2}\right)$.

With $E=\iint W(\mathbf{r}, \mathbf{q})$ drd $\mathbf{r}$ denoting the total energy of the optical signal, the central second-order moments of the Wigner distribution are defined as [8]

$$
\begin{aligned}
\mathbf{M}= & \frac{1}{E} \iint\left[\begin{array}{l}
\mathbf{r} \\
\mathbf{q}
\end{array}\right]\left[\mathbf{r}^{t}, \mathbf{q}^{t}\right] W(\mathbf{r}, \mathbf{q}) \mathrm{d} \mathbf{r} \mathrm{d} \mathbf{q} \\
= & \frac{1}{E} \iiint \int\left[\begin{array}{llll}
x x & x y & x u & x v \\
x y & y y & y u & y v \\
x u & y u & u u & u v \\
x v & y v & u v & v v
\end{array}\right] \\
& \times W(x, y, u, v) \mathrm{d} x \mathrm{~d} y \mathrm{~d} u \mathrm{~d} v
\end{aligned}
$$

and constitute a positive-definite real symmetric $4 \times 4$ moment matrix $\mathbf{M}$,

$$
\mathbf{M}=\left[\begin{array}{llll}
m_{x x} & m_{x y} & m_{x u} & m_{x v} \\
m_{x y} & m_{y y} & m_{y u} & m_{y v} \\
m_{x u} & m_{y u} & m_{u u} & m_{u v} \\
m_{x v} & m_{y v} & m_{u v} & m_{v v}
\end{array}\right]=:\left[\begin{array}{ll}
\mathbf{M}_{\mathbf{r r}} & \mathbf{M}_{\mathbf{r q}} \\
\mathbf{M}_{\mathbf{r q}}^{t} & \mathbf{M}_{\mathbf{q q}}
\end{array}\right]
$$

with ten degrees of freedom. We have chosen the origin of the coordinate system such that the first-order moments vanish: $\iint \mathbf{r} W(\mathbf{r}, \mathbf{q}) \mathrm{d} \mathbf{r} \mathrm{d} \mathbf{q}=\iint \mathbf{q} W(\mathbf{r}, \mathbf{q}) \mathrm{d} \mathbf{r} \mathrm{d} \mathbf{q}=\mathbf{0}$.

\section{FIRST-ORDER OPTICAL SYSTEM AND ITS SYMPLECTIC RAY TRANSFORMATION MATRIX}

To study the propagation of an optical signal (and in particular its moments) through a first-order optical system, we need to know how its Wigner distribution is transformed by such a system. We recall that a lossless firstorder optical system [12] can be described by its real symplectic $4 \times 4$ ray transformation matrix $\mathbf{T}$, which relates the position vector $\mathbf{r}_{i}$ and direction vector $\mathbf{q}_{i}$ of an incoming ray to the position vector $\mathbf{r}_{o}$ and direction vector $\mathbf{q}_{o}$ of the outgoing one:

$$
\left[\begin{array}{l}
\mathbf{r}_{o} \\
\mathbf{q}_{o}
\end{array}\right]=\mathbf{T}\left[\begin{array}{l}
\mathbf{r}_{i} \\
\mathbf{q}_{i}
\end{array}\right]=\left[\begin{array}{ll}
\mathbf{A} & \mathbf{B} \\
\mathbf{C} & \mathbf{D}
\end{array}\right]\left[\begin{array}{l}
\mathbf{r}_{i} \\
\mathbf{q}_{i}
\end{array}\right]
$$

Symplecticity of the matrix $\mathbf{T}$ is defined as

$$
\begin{aligned}
\mathbf{T}= & {\left[\begin{array}{ll}
\mathbf{A} & \mathbf{B} \\
\mathbf{C} & \mathbf{D}
\end{array}\right]=\left[\begin{array}{cc}
\mathbf{D}^{t} & -\mathbf{B}^{t} \\
-\mathbf{C}^{t} & \mathbf{A}^{t}
\end{array}\right]^{-1}, } \\
& \text { or } \quad \mathbf{T}^{-1}=\mathbf{J T}^{t} \mathbf{J} \quad \text { with } \quad \mathbf{J}=i\left[\begin{array}{cc}
\mathbf{0} & -\mathbf{I} \\
\mathbf{I} & \mathbf{0}
\end{array}\right]=\mathbf{J}^{-1} .
\end{aligned}
$$

From the definitions of the Wigner distribution [Eq. (1)], its moments [Eq. (2)], and the ray transformation matrix [Eq. (4)], we easily derive that when an optical signal propagates through a first-order optical system, its input and output Wigner distributions are related as [7] $W_{o}(\mathbf{A r}+\mathbf{B q}, \mathbf{C r}+\mathbf{D q})=W_{i}(\mathbf{r}, \mathbf{q})$, and its moments in the input and output planes are related as [8]

$$
\begin{aligned}
\mathbf{M}_{o} & =\left[\begin{array}{ll}
\mathbf{M}_{\mathbf{r r}} & \mathbf{M}_{\mathbf{r q}} \\
\mathbf{M}_{\mathbf{r q}}^{t} & \mathbf{M}_{\mathbf{q q}}
\end{array}\right]_{o}=\left[\begin{array}{ll}
\mathbf{A} & \mathbf{B} \\
\mathbf{C} & \mathbf{D}
\end{array}\right]\left[\begin{array}{ll}
\mathbf{M}_{\mathbf{r r}} & \mathbf{M}_{\mathbf{r q}} \\
\mathbf{M}_{\mathbf{r q}}^{t} & \mathbf{M}_{\mathbf{q q}}
\end{array}\right]_{i}\left[\begin{array}{ll}
\mathbf{A} & \mathbf{B} \\
\mathbf{C} & \mathbf{D}
\end{array}\right]^{t} \\
& =\mathbf{T M}_{i} \mathbf{T}^{t} .
\end{aligned}
$$

This input-output relation will be the basis for our further analysis.

\section{CANONICAL FORM $\triangle$ OF THE MOMENT MATRIX M}

According to Williamson's theorem [10,11], for any positive-definite real symmetric $4 \times 4$ matrix $\mathbf{M}$, there exists a real symplectic matrix $\mathbf{T}^{\circ}$ such that

$$
\mathbf{M}=\mathbf{T}^{\mathrm{o}} \boldsymbol{\Delta} \mathbf{T}^{\mathrm{o} t},
$$

cf. Eq. (6), where $\Delta$ takes the canonical form

$$
\boldsymbol{\Delta}=\left[\begin{array}{cc}
\boldsymbol{\Lambda} & \mathbf{0} \\
\mathbf{0} & \boldsymbol{\Lambda}
\end{array}\right] \text { with } \boldsymbol{\Lambda}=\left[\begin{array}{cc}
\lambda_{x} & 0 \\
0 & \lambda_{y}
\end{array}\right] \text { and } \lambda_{x} \geqslant \lambda_{y}>0
$$

From the input-output relation (7), written in the form $\mathbf{M}\left(\mathbf{T}^{\mathrm{o} t}\right)^{-1}=\mathbf{T}^{\mathrm{o}} \boldsymbol{\Delta}$, and the symplecticity condition (5), hence $\left(\mathbf{T}^{\mathrm{o} t}\right)^{-1}=\mathbf{J} \mathbf{T}^{\mathrm{o}} \mathbf{J}$, we easily derive [8] $(\mathbf{M J}) \mathbf{T}^{\mathrm{o}}=\mathbf{T}^{\mathrm{o}}(\boldsymbol{\Delta} \mathbf{J})$, and we conclude that the diagonal matrix $\Delta$ follows directly from the eigenvalues $\pm \lambda_{x}$ and $\pm \lambda_{y}$ of the permutated moment matrix $\mathbf{M J}$, and that $\mathbf{T}^{0}$ follows from the eigenvectors of $(\mathbf{M J})^{2}$ :

$$
(\mathbf{M J})^{2} \mathbf{T}^{\mathbf{o}}=\mathbf{T}^{\mathbf{o}} \boldsymbol{\Delta}^{2} .
$$

As a matter of fact, the determination of the canonical eigenvalues $\lambda_{x}$ and $\lambda_{y}$ is easy: from the two moment invariants

$$
\begin{aligned}
\lambda_{x} \lambda_{y}= & \sqrt{\operatorname{det} \mathbf{M}}=: I_{1}, \\
\lambda_{x}^{2}+\lambda_{y}^{2}= & \left(m_{x x} m_{u u}-m_{x u}^{2}\right)+\left(m_{y y} m_{v v}-m_{y v}^{2}\right) \\
& +2\left(m_{x y} m_{u v}-m_{x v} m_{y u}\right)=: I_{2},
\end{aligned}
$$

we get immediately

$$
\left(\lambda_{x} \pm \lambda_{y}\right)^{2}=I_{2} \pm 2 I_{1}
$$

and thus 


$$
\lambda_{x, y}=\frac{1}{2}\left(\sqrt{I_{2}+2 I_{1}} \pm \sqrt{I_{2}-2 I_{1}}\right) .
$$

Note that $I_{2} / 2 I_{1}=\frac{1}{2}\left(\lambda_{x} / \lambda_{y}+\lambda_{y} / \lambda_{x}\right) \geqslant 1$ and that the equality sign arises for $\lambda_{x}=\lambda_{y}$. The equality of $\lambda_{x}$ and $\lambda_{y}$ corresponds to the intrinsically isotropic case, while $\lambda_{x} \neq \lambda_{y}$ corresponds to the anisotropic case [10].

\section{MODIFIED IWASAWA DECOMPOSITION AND ORTHOSYMPLECTIC SYSTEMS}

To analyze how a moment matrix $\mathbf{M}$ can be brought into its canonical form $\Delta$, we represent the ray transformation matrix $\mathbf{T}^{\circ}$ that appears in Eq. (7) in its modified Iwasawa decomposition [10,13-16]. We write

$$
\begin{aligned}
\mathbf{T}^{\mathbf{o}} & =\left[\begin{array}{ll}
\mathbf{A} & \mathbf{B} \\
\mathbf{C} & \mathbf{D}
\end{array}\right]=\left[\begin{array}{cc}
\mathbf{I} & \mathbf{0} \\
-\mathbf{L} & \mathbf{I}
\end{array}\right]\left[\begin{array}{cc}
\mathbf{S} & \mathbf{0} \\
\mathbf{0} & \mathbf{S}^{-1}
\end{array}\right]\left[\begin{array}{cc}
\mathbf{X} & \mathbf{Y} \\
-\mathbf{Y} & \mathbf{X}
\end{array}\right] \\
& =\mathbf{T}_{l}(\mathbf{L}) \mathbf{T}_{m}(\mathbf{S}) \mathbf{T}_{o}(\mathbf{X}+i \mathbf{Y}),
\end{aligned}
$$

in which cascade the first matrix, $\mathbf{T}_{l}(\mathbf{L})$, corresponds to an anamorphic lens $\mathcal{L}(\mathbf{L})$ described by the symmetric matrix

$$
\mathbf{L}=-\left(\mathbf{C A}^{t}+\mathbf{D B} \mathbf{B}^{t}\right)\left(\mathbf{A} \mathbf{A}^{t}+\mathbf{B B}^{t}\right)^{-1}=\mathbf{L}^{t},
$$

the second matrix, $\mathbf{T}_{m}(\mathbf{S})$, corresponds to a magnifier $\mathcal{M}(\mathbf{S})$ described by the positive-definite symmetric matrix

$$
\mathbf{S}=\left(\mathbf{A} \mathbf{A}^{t}+\mathbf{B} \mathbf{B}^{t}\right)^{1 / 2}=\mathbf{S}^{t},
$$

and the third matrix, $\mathbf{T}_{o}(\mathbf{X}+i \mathbf{Y})$, corresponds to a socalled orthosymplectic system $\mathcal{O}(\mathbf{U})$ described by the unitary matrix

$$
\mathbf{U}=\mathbf{X}+i \mathbf{Y}=\left(\mathbf{A A}^{t}+\mathbf{B B}^{t}\right)^{-1 / 2}(\mathbf{A}+i \mathbf{B})=\left(\mathbf{U}^{\dagger}\right)^{-1},
$$

where the superscript ${ }^{\dagger}$ represents the combined action of conjugation and transposition: $\mathbf{U}^{\dagger}=\left(\mathbf{U}^{*}\right)^{t}$. Note that the ray transformation matrix $\mathbf{T}_{o}$ of an orthosymplectic system is not only symplectic, but also orthogonal: $\mathbf{T}_{o}^{-1}$ $=\mathbf{J T}_{o}^{t} \mathbf{J}=\mathbf{T}_{o}^{t}$.

Any first-order optical system can thus be realized by means of the cascade $\mathcal{L}(\mathbf{L}) \mathcal{M}(\mathbf{S}) \mathcal{O}(\mathbf{U})$. In Section 6 we will concentrate on the orthosymplectic part $\mathcal{O}(\mathbf{U})$ and study its effect when it operates on a beam whose moment matrix is in the canonical form $\Delta$. As a preparation, we recall some basic properties of orthosymplectic systems.

Three basic orthosymplectic systems are: the separable fractional Fourier transformer $\mathcal{F}\left(\gamma_{1}, \gamma_{2}\right)$, the rotator $\mathcal{R}(\phi)$, and the gyrator $\mathcal{G}(\phi)$, defined by the unitary matrices

$$
\begin{aligned}
\mathbf{U}_{f}\left(\gamma_{1}, \gamma_{2}\right) & =\left[\begin{array}{cc}
\exp \left(i \gamma_{1}\right) & 0 \\
0 & \exp \left(i \gamma_{2}\right)
\end{array}\right], \\
\mathbf{U}_{r}(\phi) & =\left[\begin{array}{cc}
\cos \phi & \sin \phi \\
-\sin \phi & \cos \phi
\end{array}\right], \\
\mathbf{U}_{g}(\phi) & =\left[\begin{array}{cc}
\cos \phi & i \sin \phi \\
i \sin \phi & \cos \phi
\end{array}\right],
\end{aligned}
$$

and with ray transformation matrices $\mathbf{T}_{f}\left(\gamma_{1}, \gamma_{2}\right), \mathbf{T}_{r}(\phi)$, $\mathbf{T}_{g}(\phi)$, respectively. These basic systems are additive in their parameters and correspond to rotations in phase space: the rotator $\mathcal{R}(\phi)$ performs a rotation through an angle $\phi$ in the $x y$ and the $u v$ planes, the gyrator $\mathcal{G}(\phi)$ in the $x u$ and $y v$ planes, and the separable fractional Fourier transformer $\mathcal{F}\left(\gamma_{1}, \gamma_{2}\right)$ in the $x u$ plane (through an angle $\gamma_{1}$ ) and the $y v$ plane (through an angle $\gamma_{2}$ ). From the many decompositions of a general orthosymplectic system $\mathcal{O}(\mathbf{U})$ into more basic ones, we mention in particular

$$
\mathcal{O}(\mathbf{U})=\mathcal{R}(-\alpha) \mathcal{G}(-\beta) \mathcal{F}\left(\gamma_{x}-\psi, \gamma_{y}+\psi\right),
$$

which follows from the identities

$$
\begin{aligned}
\mathbf{U} & =\left[\begin{array}{cc}
\exp \left(i \gamma_{x}\right) \cos \phi & -\exp \left[i\left(\gamma_{y}+\gamma\right)\right] \sin \phi \\
\exp \left[i\left(\gamma_{x}-\gamma\right)\right] \sin \phi & \exp \left(i \gamma_{y}\right) \cos \phi
\end{array}\right] \\
& =\mathbf{U}_{f}\left(\frac{1}{2} \gamma,-\frac{1}{2} \gamma\right) \mathbf{U}_{r}(-\phi) \mathbf{U}_{f}\left(-\frac{1}{2} \gamma, \frac{1}{2} \gamma\right) \mathbf{U}_{f}\left(\gamma_{x}, \gamma_{y}\right) \\
& =\mathbf{U}_{r}(-\alpha) \mathbf{U}_{g}(-\beta) \mathbf{U}_{f}(-\psi, \psi) \mathbf{U}_{f}\left(\gamma_{x}, \gamma_{y}\right),
\end{aligned}
$$

where the angles $\alpha$ and $\beta$ are related to $\gamma$ and $\phi$ by

$$
\begin{aligned}
& \sin 2 \beta=\sin 2 \phi \sin \gamma, \\
& \cos 2 \phi=\cos 2 \alpha \cos 2 \beta,
\end{aligned}
$$

and $\psi$ follows from $\tan \psi=\tan \alpha \tan \beta$.

\section{GENERALIZED CANONICAL FORM $\mathbf{M}^{\mathbf{0}}$}

Let us consider a moment matrix $\mathbf{M}^{0}$ that can be brought into its canonical form $\Delta$ by means of an orthosymplectic system $\mathcal{O}(\mathbf{U})$ :

$$
\begin{aligned}
& \mathbf{M}^{0}=\mathbf{T}_{o} \Delta \mathbf{T}_{o}^{t}=\left[\begin{array}{cc}
\mathbf{X} & \mathbf{Y} \\
-\mathbf{Y} & \mathbf{X}
\end{array}\right]\left[\begin{array}{cc}
\boldsymbol{\Lambda} & \mathbf{0} \\
\mathbf{0} & \boldsymbol{\Lambda}
\end{array}\right]\left[\begin{array}{cc}
\mathbf{X}^{t} & -\mathbf{Y}^{t} \\
\mathbf{Y}^{t} & \mathbf{X}^{t}
\end{array}\right] \\
& =\left[\begin{array}{cc}
\mathbf{X} \Lambda \mathbf{X}^{t}+\mathbf{Y} \Lambda \mathbf{Y}^{t} & -\mathbf{X} \mathbf{\Lambda} \mathbf{Y}^{t}+\mathbf{Y} \Lambda \mathbf{X}^{t} \\
\mathbf{X} \Lambda \mathbf{Y}^{t}-\mathbf{Y} \Lambda \mathbf{X}^{t} & \mathbf{X} \mathbf{\Lambda} \mathbf{X}^{t}+\mathbf{Y} \Lambda \mathbf{Y}^{t}
\end{array}\right] \\
& =:\left[\begin{array}{cc}
\mathbf{M}_{\mathbf{r r}}^{\mathrm{o}} & \mathbf{M}_{\mathbf{r q}}^{\mathrm{o}} \\
\left(\mathbf{M}_{\mathbf{r q}}^{\mathrm{o}}\right)^{t} & \mathbf{M}_{\mathbf{q q}}^{\mathrm{o}}
\end{array}\right]=\left[\begin{array}{cc}
\mathbf{M}_{\mathbf{r r}}^{\mathrm{o}} & \mathbf{M}_{\mathbf{r q}}^{\mathrm{o}} \\
-\mathbf{M}_{\mathbf{r q}}^{\mathrm{o}} & \mathbf{M}_{\mathbf{r r}}^{\mathrm{o}}
\end{array}\right] \text {. }
\end{aligned}
$$

The moment matrix $\mathbf{M}^{\mathrm{o}}$ will be called the generalized canonical form. Note that $\mathbf{M}_{\mathbf{q q}}^{\mathrm{o}}=\mathbf{M}_{\mathbf{r r}}^{\mathrm{o}}$ and $\left(\mathbf{M}_{\mathbf{r q}}^{\mathrm{o}}\right)^{t}=-\mathbf{M}_{\mathbf{r q}}^{\mathrm{o}}$, that the moment matrix $\mathbf{M}^{0}$ is completely described by the expression $\mathbf{M}_{\mathbf{r r}}^{\mathrm{o}}+i \mathbf{M}_{\mathbf{r q}}^{\mathrm{o}}$, and that Eq. (18) can equivalently be written in the form

$$
\mathbf{M}_{\mathbf{r r}}^{\mathrm{o}}+i \mathbf{M}_{\mathbf{r q}}^{\mathrm{o}}=\mathbf{U} \boldsymbol{\Lambda} \mathbf{U}^{\dagger}
$$

In the intrinsically isotropic case [10], with $\lambda_{x}=\lambda_{y}=\lambda$, we have $\mathbf{M}_{\mathbf{r r}}^{\mathrm{o}}+i \mathbf{M}_{\mathbf{r q}}^{\mathrm{o}}=\lambda \mathbf{I}$, and thus $\mathbf{M}_{\mathbf{r r}}^{\mathrm{o}}=\lambda \mathbf{I}$ and $\mathbf{M}_{\mathbf{r q}}^{\mathrm{o}}=\mathbf{0}$; hence, the moment matrix $\mathbf{M}^{0}=\boldsymbol{\Delta}=\lambda \mathbf{I}$ is a scalar matrix.

For a separable fractional Fourier transformer $\mathcal{F}\left(\gamma_{1}, \gamma_{2}\right)$, whose unitary representation $\mathbf{U}_{f}\left(\gamma_{1}, \gamma_{2}\right)$ is diagonal, Eq. (19) leads to $\mathbf{M}_{\mathbf{r r}}^{\mathrm{o}}+i \mathbf{M}_{\mathbf{r q}}^{\mathrm{o}}=\boldsymbol{\Lambda}$, and we conclude that such a system has no effect on the moments. In the basic cascade (15), we can thus ignore the separable fractional Fourier transformer $\mathcal{F}\left(\gamma_{x}-\psi, \gamma_{y}+\psi\right)$, and we can restrict ourselves to the subsystem $\mathcal{R}(-\alpha) \mathcal{G}(-\beta)$. The generalized canonical form can then be generated by letting the cascade of a rotator and a gyrator, $\mathcal{R}(-\alpha) \mathcal{G}(-\beta)$, operate upon the canonical form, in which case Eq. (19) yields

$$
\mathbf{M}_{\mathbf{r r}}^{\mathrm{o}}+i \mathbf{M}_{\mathbf{r q}}^{\mathrm{o}}=\mathbf{U}_{r}(-\alpha) \mathbf{U}_{g}(-\beta) \boldsymbol{\Lambda} \mathbf{U}_{g}(\beta) \mathbf{U}_{r}(\alpha) .
$$

Equation (20) leads in detail to the expressions 


$$
\begin{aligned}
m_{x x}^{o}+m_{y y}^{o} & =\lambda_{x}+\lambda_{y}, \\
m_{x x}^{o}-m_{y y}^{o} & =\left(\lambda_{x}-\lambda_{y}\right) \cos 2 \beta \cos 2 \alpha, \\
2 m_{x y}^{o} & =\left(\lambda_{x}-\lambda_{y}\right) \cos 2 \beta \sin 2 \alpha, \\
2 m_{x v}^{o} & =\left(\lambda_{x}-\lambda_{y}\right) \sin 2 \beta,
\end{aligned}
$$

and also $m_{x u}^{0}=m_{y v}^{0}=m_{y u}^{0}+m_{x v}^{0}=0$. Note that $\mathbf{M}^{0}$ has only four degrees of freedom.

\section{REPRESENTATION OF THE GENERALIZED CANONICAL FORM $\mathrm{M}^{\circ} \mathrm{ON}$ THE ANGULAR POINCARE SPHERE}

Let us now represent the generalized canonical form of a beam (i.e., with a moment matrix of the form $\mathbf{M}^{\circ}$ ) on the angular Poincaré sphere. With

$$
\begin{aligned}
& Q_{1}=m_{x x}^{0}-m_{y y}^{0}=Q \cos 2 \beta \cos 2 \alpha, \\
& Q_{2}=2 m_{x y}^{0}=Q \cos 2 \beta \sin 2 \alpha, \\
& Q_{3}=2 m_{x v}^{0}=Q \sin 2 \beta \\
& Q=\sqrt{Q_{1}^{2}+Q_{2}^{2}+Q_{3}^{2}}=\lambda_{x}-\lambda_{y},
\end{aligned}
$$

and with $\left[Q_{1}, Q_{2}, Q_{3}\right]$ representing the components of the orbital angular momentum [2,4], the canonical form $\Delta$, for which $\mathbf{M}^{0}$ satisfies the additional condition $m_{x y}^{0}=m_{x v}^{0}=0$, can be represented on the angular Poincaré sphere $[2,4]$ with radius $Q$ and located at the point $\left[Q_{1}, Q_{2}, Q_{3}\right]$ $=[Q, 0,0]$, which is the point where the equator (in the plane $Q_{3}=0$ ) and the main meridian (in the plane $Q_{2}=0$ ) cross. From Eqs. (21b)-(21d) we conclude that the general cascade $\mathcal{R}(-\alpha) \mathcal{G}(-\beta)$ yields

$$
\left[Q_{1}, Q_{2}, Q_{3}\right]=[Q \cos 2 \beta \cos 2 \alpha, Q \cos 2 \beta \sin 2 \alpha, Q \sin 2 \beta],
$$

and that, starting from the point $[Q, 0,0]$, the entire sphere can thus be populated: the gyrator $\mathcal{G}(-\beta)$ brings us from $[Q, 0,0]$ to the point $[Q \cos 2 \beta, 0, Q \sin 2 \beta]$ on the main meridian with the correct latitude $2 \beta$, and the subsequent rotator $\mathcal{R}(-\alpha)$ then brings us to the correct longitude $2 \alpha$. We conclude that the canonical form of a beam, located at $[Q, 0,0]$ on the Poincaré sphere, can be considered as a generator for beams with the same value of $Q$ but with different orientations of the orbital angular momentum.

\section{CONJUGATE CANONICAL FORM $\overline{\mathbf{M}}^{\mathrm{o}}(\boldsymbol{\beta})$}

As a consequence of the results of the previous section, the system with ray transformation matrix $\mathbf{T}^{0}$ can be reduced to $\mathcal{L}(\mathbf{L}) \mathcal{M}(\mathbf{S}) \mathcal{R}(-\alpha) \mathcal{G}(-\beta)$, and we remark that the subsystem $\mathcal{L}(\mathbf{L}) \mathcal{M}(\mathbf{S}) \mathcal{R}(-\alpha)$ is a system that operates between conjugate planes. Based on this observation, we introduce the conjugate canonical form $\overline{\mathbf{M}}^{\mathrm{o}}(\beta)$,

$$
\overline{\mathbf{M}}^{\mathrm{o}}(\beta)=\left[\begin{array}{cc}
\overline{\mathbf{M}}_{\mathbf{r r}}^{\mathrm{o}}(\beta) & \overline{\mathbf{M}}_{\mathbf{r q}}^{\mathrm{o}}(\beta) \\
-\overline{\mathbf{M}}_{\mathbf{r q}}^{\mathrm{o}}(\beta) & \overline{\mathbf{M}}_{\mathbf{r r}}^{\mathrm{o}}(\beta)
\end{array}\right],
$$

which arises when we let the gyrator $\mathcal{G}(-\beta)$ operate upon the canonical form $\Delta$ and which is located on the main meridian $\left(Q_{2}=0\right)$ of the Poincaré sphere. We have

$$
\overline{\mathbf{M}}_{\mathbf{r r}}^{\mathrm{o}}(\beta)+i \overline{\mathbf{M}}_{\mathbf{r q}}^{\mathrm{o}}(\beta)=\mathbf{U}_{g}(-\beta) \boldsymbol{\Lambda} \mathbf{U}_{g}(\beta),
$$

cf. Eq. (20), and thus

$$
\begin{gathered}
\bar{m}_{x x}^{o}=\lambda_{x} \cos ^{2} \beta+\lambda_{y} \sin ^{2} \beta, \\
\bar{m}_{y y}^{o}=\lambda_{x} \sin ^{2} \beta+\lambda_{y} \cos ^{2} \beta, \\
2 \bar{m}_{x v}^{o}=\left(\lambda_{x}-\lambda_{y}\right) \sin 2 \beta=2 m_{x v}^{o} .
\end{gathered}
$$

Note that $\bar{m}_{x y}^{o}=0$ (and $\bar{m}_{x u}^{o}=\bar{m}_{y v}^{o}=\bar{m}_{y u}^{o}+\bar{m}_{x v}^{o}=0$ ) and that $\overline{\mathbf{M}}^{\mathrm{o}}(\beta)$ has only three degrees of freedom. We remark that

$$
\operatorname{det} \overline{\mathbf{M}}_{\mathbf{r r}}^{\mathrm{o}}(\beta)=\left[\frac{1}{2}\left(\lambda_{x}-\lambda_{y}\right) \sin 2 \beta\right]^{2}+\lambda_{x} \lambda_{y}
$$

and that it takes its maximum value $\frac{1}{4}\left(\lambda_{x}+\lambda_{y}\right)^{2}$ for $\beta$ $= \pm \frac{1}{4} \pi$.

Any moment matrix $\mathbf{M}$ can be brought into its conjugate canonical form $\overline{\mathbf{M}}^{\mathrm{o}}(\beta)$ by the cascade of a lens $\mathcal{L}(\mathbf{L})$, a magnifier $\mathcal{M}(\mathbf{S})$, and a rotator $\mathcal{R}(-\alpha)$. We write

$$
\begin{aligned}
\mathbf{M}= & {\left[\begin{array}{ll}
\mathbf{M}_{\mathbf{r r}} & \mathbf{M}_{\mathbf{r q}} \\
\mathbf{M}_{\mathbf{r q}}^{t} & \mathbf{M}_{\mathbf{q q}}
\end{array}\right]=\left[\begin{array}{cc}
\mathbf{I} & \mathbf{0} \\
-\mathbf{L} & \mathbf{I}
\end{array}\right] \mathbf{M}^{\mathbf{L}}\left[\begin{array}{cc}
\mathbf{I} & -\mathbf{L} \\
\mathbf{0} & \mathbf{I}
\end{array}\right], } \\
\mathbf{M}^{\mathbf{L}}= & {\left[\begin{array}{cc}
\mathbf{S} & \mathbf{0} \\
\mathbf{0} & \mathbf{S}^{-1}
\end{array}\right]\left[\begin{array}{cc}
\mathbf{U}_{r}(-\alpha) & \mathbf{0} \\
\mathbf{0} & \mathbf{U}_{r}(-\alpha)
\end{array}\right] \overline{\mathbf{M}}^{\mathrm{o}}(\beta) } \\
& \times\left[\begin{array}{cc}
\mathbf{U}_{r}(\alpha) & \mathbf{0} \\
\mathbf{0} & \mathbf{U}_{r}(\alpha)
\end{array}\right]\left[\begin{array}{cc}
\mathbf{S} & \mathbf{0} \\
\mathbf{0} & \mathbf{S}^{-1}
\end{array}\right] \\
= & {\left[\begin{array}{cc}
\mathbf{M}_{\mathbf{r r}}^{\mathbf{L}} & \mathbf{M}_{\mathbf{r q}}^{\mathbf{L}} \\
\left(\mathbf{M}_{\mathbf{r q}}^{\mathbf{L}}\right)^{t} & \mathbf{M}_{\mathbf{q q}}^{\mathbf{L}}
\end{array}\right], }
\end{aligned}
$$

and thus

$$
\begin{aligned}
& \mathbf{M}_{\mathbf{r r}}^{\mathbf{L}}=\mathbf{S U}_{r}(-\alpha) \overline{\mathbf{M}}_{\mathbf{r r}}^{0}(\beta) \mathbf{U}_{r}(\alpha) \mathbf{S}=\mathbf{M}_{\mathbf{r r}}, \\
& \mathbf{M}_{\mathbf{q q}}^{\mathbf{L}}=\mathbf{S}^{-1} \mathbf{U}_{r}(-\alpha) \overline{\mathbf{M}}_{\mathbf{r r}}^{0}(\beta) \mathbf{U}_{r}(\alpha) \mathbf{S}^{-1} \\
& \mathbf{M}_{\mathbf{r q}}^{\mathbf{L}}=\mathbf{S U}_{r}(-\alpha) \overline{\mathbf{M}}_{\mathbf{r q}}^{0}(\beta) \mathbf{U}_{r}(\alpha) \mathbf{S}^{-1} .
\end{aligned}
$$

From Eqs. (28a) and (28b) we get the relationship

$$
\mathbf{S}^{-1} \mathbf{M}_{\mathbf{r r}} \mathbf{S}^{-1}=\mathbf{S} \mathbf{M}_{\mathbf{q q}}^{\mathbf{L}} \mathbf{S}=\mathbf{U}_{r}(-\alpha) \overline{\mathbf{M}}_{\mathbf{r r}}^{\mathrm{o}}(\beta) \mathbf{U}_{r}(\alpha),
$$

which will play an important role later, and we also note that

$$
\operatorname{det} \mathbf{M}_{\mathbf{r r}}=(\operatorname{det} \mathbf{S})^{2} \operatorname{det} \overline{\mathbf{M}}_{\mathbf{r r}}^{\mathrm{o}}(\beta) \text {. }
$$

We finally remark that the rotator $\mathcal{R}(-\alpha)$ in the cascade $\mathcal{L}(\mathbf{L}) \mathcal{M}(\mathbf{S}) \mathcal{R}(-\alpha)$ may be shifted to the other end of the cascade, leading to $\mathcal{R}(-\alpha) \mathcal{L}(\widetilde{\mathbf{L}}) \mathcal{M}(\widetilde{\mathbf{S}})$, with the original 
lens and magnifier rotated through the angle $\alpha$ : $\widetilde{\mathbf{L}}$ $=\mathbf{U}_{r}(\alpha) \mathbf{L U}_{r}(-\alpha)$ and $\widetilde{\mathbf{S}}=\mathbf{U}_{r}(\alpha) \mathbf{S} \mathbf{U}_{r}(-\alpha)$. From this observation we conclude that-unlike the latitude- the longitude of the generalized canonical form on the Poincaré sphere does not have a strong physical meaning: it is solely determined by the choice of the transverse coordinate system, i.e., by the actual orientations of the $x$ and $y$ axes. A rotation of the coordinate system around the $z$ axis corresponds to a rotation of the Poincaré sphere around the polar axis.

\section{A. One-Dimensional Case}

In the one-dimensional case,

$$
\begin{aligned}
\mathbf{M}= & {\left[\begin{array}{ll}
m_{x x} & m_{x u} \\
m_{x u} & m_{u u}
\end{array}\right]=\left[\begin{array}{cc}
1 & 0 \\
-\ell & 1
\end{array}\right]\left[\begin{array}{cc}
s & 0 \\
0 & s^{-1}
\end{array}\right]\left[\begin{array}{cc}
\lambda & 0 \\
0 & \lambda
\end{array}\right]\left[\begin{array}{cc}
s & 0 \\
0 & s^{-1}
\end{array}\right] } \\
& \times\left[\begin{array}{cc}
1 & -\ell \\
0 & 1
\end{array}\right]=\lambda\left[\begin{array}{cc}
s^{2} & -s^{2} \ell \\
-s^{2} \ell & s^{-2}-s^{2} \ell^{2}
\end{array}\right]
\end{aligned}
$$

with $\mathbf{T}^{\circ}$ realized by a lens $\mathcal{L}(\ell)$ and a magnifier $\mathcal{M}(s)$, we easily derive that $\lambda=\sqrt{\operatorname{det} \mathbf{M}}=\bar{m}_{x x}^{0}, s^{2}=m_{x x} / \lambda$, and $-\ell$ $=m_{x u} / m_{x x}$. Note that in the one-dimensional case, the conjugate canonical form $\overline{\mathbf{M}}^{0}$ is automatically in the canonical form: $\overline{\mathbf{M}}^{\mathrm{o}}=\boldsymbol{\Delta}$.

\section{B. Intrinsically Isotropic Case}

The intrinsically isotropic case $\lambda_{x}=\lambda_{y}=\lambda$ strongly resembles the one-dimensional case. We then have $\Lambda=\lambda \mathbf{I}$, the orthosymplectic subsystem $\mathcal{R}(-\alpha) \mathcal{G}(-\beta)$ is now irrelevant, and the moment matrix $\mathbf{M}$ can be expressed as

$$
\begin{aligned}
\mathbf{M}= & {\left[\begin{array}{ll}
\mathbf{M}_{\mathbf{r r}} & \mathbf{M}_{\mathbf{r q}} \\
\mathbf{M}_{\mathbf{r q}}^{t} & \mathbf{M}_{\mathbf{q q}}
\end{array}\right]=\left[\begin{array}{cc}
\mathbf{I} & \mathbf{0} \\
-\mathbf{L} & \mathbf{I}
\end{array}\right]\left[\begin{array}{cc}
\mathbf{S} & \mathbf{0} \\
\mathbf{0} & \mathbf{S}^{-1}
\end{array}\right]\left[\begin{array}{cc}
\lambda \mathbf{I} & \mathbf{0} \\
\mathbf{0} & \lambda \mathbf{I}
\end{array}\right]\left[\begin{array}{cc}
\mathbf{S} & \mathbf{0} \\
\mathbf{0} & \mathbf{S}^{-1}
\end{array}\right] } \\
& \times\left[\begin{array}{cc}
\mathbf{I} & -\mathbf{L} \\
\mathbf{0} & \mathbf{I}
\end{array}\right]=\lambda\left[\begin{array}{cc}
\mathbf{S}^{2} & -\mathbf{S}^{2} \mathbf{L} \\
-\mathbf{L} \mathbf{S}^{2} & \mathbf{S}^{-2}-\mathbf{L} \mathbf{S}^{2} \mathbf{L}
\end{array}\right],
\end{aligned}
$$

with $\mathbf{S}^{2}=\lambda^{-1} \mathbf{M}_{\mathbf{r r}}$ and $-\mathbf{L}=\mathbf{M}_{\mathbf{r r}}^{-1} \mathbf{M}_{\mathbf{r q}}$. We remark that the matrix $\lambda^{-1} \mathbf{M}$ is symplectic and we recall that when a symplectic moment matrix $\mathbf{M}$ propagates through an ABCD system, its symplectic character is preserved, the proportionality factor $\lambda$ remains the same, and $-\mathbf{L} \pm i \mathbf{S}^{-2}$ $=\mathbf{M}_{\mathbf{r r}}^{-1}\left(\mathbf{M}_{\mathbf{r q}} \pm i \lambda \mathbf{I}\right)=: \mathbf{Z}^{ \pm}$propagates according to the bilinear ABCD law [8]

$$
\mathbf{Z}_{o}^{ \pm}=\left(\mathbf{C}+\mathbf{D} \mathbf{Z}_{i}^{ \pm}\right)\left(\mathbf{A}+\mathbf{B} \mathbf{Z}_{i}^{ \pm}\right)^{-1}
$$

The latter propagation law is an easy equivalent for the input-output relation (6).

To see the strong resemblance between the intrinsically isotropic case and the one-dimensional case, we remark that the relations $\mathbf{M}_{\mathbf{r r}}^{-1} \mathbf{M}_{\mathbf{r q}}=-\mathbf{L}$ and $\lambda^{-1} \mathbf{M}_{\mathbf{r r}}=\mathbf{S}^{2}$ are in complete analogy with $m_{x x}^{-1} m_{x u}=-\ell$ and $\lambda^{-1} m_{x x}=s^{2}$. Note that in the intrinsically isotropic case, with seven degrees of freedom, everything is determined by $\operatorname{det} \mathbf{M}$ and the submatrices $\mathbf{M}_{\mathbf{r r}}$ and $\mathbf{M}_{\mathbf{r q}}$; the submatrix $\mathbf{M}_{\mathbf{q q}}$ follows as $\lambda^{2} \mathbf{M}_{\mathbf{r r}}^{-1}+\mathbf{M}_{\mathbf{r r}}^{-1} \mathbf{M}_{\mathbf{r g}}^{2}$, which is again in analogy with $m_{u u}$ $=\lambda^{2} m_{x x}^{-1}+m_{x x}^{-1} m_{x u}^{2}$ in the one-dimensional case.
C. Two-Dimensional Case with $\boldsymbol{\beta}=\mathbf{0}$

Before we study the general two-dimensional case in Section 10 , we first consider the special case $\beta=0$, for which $\overline{\mathbf{M}}_{\mathbf{r r}}^{o}(\beta)=\boldsymbol{\Lambda}$ and $\overline{\mathbf{M}}_{\mathbf{r q}}^{\mathrm{o}}(\beta)=\mathbf{0}$. The conjugate canonical form $\overline{\mathbf{M}}^{\mathrm{o}}(0)$ is again automatically in the canonical form $\Delta$, and for the moment matrix $\mathbf{M}$, which now has nine degrees of freedom, we easily verify via Eqs. (27a) and (27b) that $\mathbf{M}_{\mathbf{r q}} \mathbf{M}_{\mathbf{r r}}=\mathbf{M}_{\mathbf{r r}} \mathbf{M}_{\mathbf{r q}}^{t}$ and that $\mathbf{M}_{\mathbf{r q}}=-\mathbf{M}_{\mathbf{r r}} \mathbf{L}$. We can now determine the lens $\mathcal{L}(\mathbf{L})$, the magnifier $\mathcal{M}(\mathbf{S})$, and the rotator $\mathcal{R}(-\alpha)$ directly from the moment matrix $\mathbf{M}$, as is shown in Appendix A.

Whereas $\mathbf{M}_{\mathbf{r q}} \mathbf{M}_{\mathbf{r r}}=\mathbf{M}_{\mathbf{r r}} \mathbf{M}_{\mathbf{r q}}^{t}$ leads to a generalized canonical form that is located on the equator of the Poincaré sphere $(\beta=0)$, this is no longer the case when $\mathbf{M}_{\mathbf{r q}} \mathbf{M}_{\mathbf{r r}}$ $\neq \mathbf{M}_{\mathbf{r r}} \mathbf{M}_{\mathbf{r q}}^{t}$. In Section 9 we will relate the latter case to the appearance of twist.

\section{TWIST AND ORBITAL ANGULAR MOMENTUM}

In the general two-dimensional case, the moment matrix $\mathbf{M}$ can be decomposed in the form

$$
\begin{aligned}
\mathbf{M}= & {\left[\begin{array}{ll}
\mathbf{M}_{\mathbf{r r}} & \mathbf{M}_{\mathbf{r q}} \\
\mathbf{M}_{\mathbf{r q}}^{t} & \mathbf{M}_{\mathbf{q q}}
\end{array}\right]=\left[\begin{array}{cc}
\mathbf{I} & \mathbf{0} \\
\mathbf{M}_{\mathbf{r q}}^{t} \mathbf{M}_{\mathbf{r r}}^{-1} & \mathbf{I}
\end{array}\right] } \\
& \times\left[\begin{array}{cc}
\mathbf{M}_{\mathbf{r r}} & \mathbf{0} \\
\mathbf{0} & \mathbf{M}_{\mathbf{q q}}-\mathbf{M}_{\mathbf{r q}}^{t} \mathbf{M}_{\mathbf{r r}}^{-1} \mathbf{M}_{\mathbf{r q}}
\end{array}\right]\left[\begin{array}{cc}
\mathbf{I} & \mathbf{M}_{\mathbf{r r}}^{-1} \mathbf{M}_{\mathbf{r q}} \\
\mathbf{0} & \mathbf{I}
\end{array}\right] .
\end{aligned}
$$

We recall that when the matrix $\mathbf{M}_{\mathbf{r r}}^{-1} \mathbf{M}_{\mathbf{r q}}$ is symmetric, which happens for $\beta=0$, it defines immediately the lens in the decomposition (27a): $\mathbf{L}=-\mathbf{M}_{\mathbf{r r}}^{-1} \mathbf{M}_{\mathbf{r q}}$.

The twist of a beam [17-24] is connected to the asymmetry of the matrix $\mathbf{M}_{\mathbf{r r}}^{-1} \mathbf{M}_{\mathbf{r q}}$. To measure the degree of twist, we use the asymmetry of the (normalized) matrix

$$
\mathbf{M}_{\mathbf{r r}}^{1 / 2}\left(\mathbf{M}_{\mathbf{r r}}^{-1} \mathbf{M}_{\mathbf{r q}}\right) \mathbf{M}_{\mathbf{r r}}^{1 / 2}=\mathbf{M}_{\mathbf{r r}}^{-1 / 2} \mathbf{M}_{\mathbf{r q}} \mathbf{M}_{\mathbf{r r}}^{1 / 2}=\mathbf{M}_{\mathbf{r r}}^{-1 / 2}\left(\mathbf{M}_{\mathbf{r q}} \mathbf{M}_{\mathbf{r r}}\right) \mathbf{M}_{\mathbf{r r}}^{-1 / 2},
$$

and we define the (normalized) twist parameter as

$$
T=\frac{\left(m_{x u}-m_{y v}\right) m_{x y}+m_{x v} m_{y y}-m_{x x} m_{y u}}{\left(\operatorname{det} \mathbf{M}_{\mathbf{r r}}\right)^{1 / 2}} .
$$

Note that the numerator in the above expression corresponds to the asymmetry of $\mathbf{M}_{\mathbf{r q}} \mathbf{M}_{\mathbf{r r}}$, i.e., to the upper offdiagonal element of $\mathbf{M}_{\mathbf{r q}} \mathbf{M}_{\mathbf{r r}}-\left(\mathbf{M}_{\mathbf{r q}} \mathbf{M}_{\mathbf{r r}}\right)^{t}$.

Another quantity that is defined by means of the second-order moments is the orbital angular momentum [25-27]

$$
\Lambda=m_{x v}-m_{y u},
$$

i.e., the asymmetry of the matrix $\mathbf{M}_{\mathbf{r q}}$. Note that in the case of a beam with a symmetrical intensity profile, i.e., $m_{x x}=m_{y y}$ and $m_{x y}=0$, the orbital angular momentum is identical to the twist, and the difference $\Lambda-T$ may thus act in general as a measure for the asymmetry of the beam.

Using Eqs. (21a)-(21d) and (25a)-(25c), we easily derive that for the generalized and conjugate canonical forms we have

$$
\Lambda^{0}=\bar{\Lambda}^{0}=2 m_{x v}^{0}=\left(\lambda_{x}-\lambda_{y}\right) \sin 2 \beta,
$$




$$
T^{o}=\bar{T}^{0}=\left(\lambda_{x}+\lambda_{y}\right) \frac{m_{x v}^{o}}{\sqrt{\left(m_{x v}^{0}\right)^{2}+\lambda_{x} \lambda_{y}}} .
$$

Considered as a function of $\beta$, the orbital angular momentum $\Lambda^{\circ}$ and the twist $T^{\circ}$ take their extremum values when $2 \beta= \pm \frac{1}{2} \pi$, i.e., at the poles of the Poincaré sphere: $\left|\Lambda^{0}\right|_{\max }=\left|T^{0}\right|_{\max }=\lambda_{x}-\lambda_{y}$. For $\beta=0$, i.e., if the generalized canonical form is located on the equator of the Poincaré sphere, the twist and the orbital angular momentum vanish.

\section{A. Propagation of the Twist between Conjugate Planes} We will prove the important property that $T$ is an invariant if the light propagates in a first-order optical system between conjugate planes. Propagation between conjugate planes can be described by the ray transformation matrix

$$
\mathbf{T}=\left[\begin{array}{cc}
\mathbf{A} & \mathbf{0} \\
-\mathbf{L A} & \left(\mathbf{A}^{t}\right)^{-1}
\end{array}\right]
$$

where $\mathbf{L}=\mathbf{L}^{t}$ is a symmetric matrix that can be associated with a lens. Using the input-output relation (6), we easily derive

$$
\begin{aligned}
\left(\mathbf{M}_{\mathbf{r r}}\right)_{o} & =\mathbf{A}\left(\mathbf{M}_{\mathbf{r r}}\right)_{i} \mathbf{A}^{t} \\
\left(\mathbf{M}_{\mathbf{r q}}\right)_{o} & =-\mathbf{A}\left(\mathbf{M}_{\mathbf{r r}}\right)_{i} \mathbf{A}^{t} \mathbf{L}+\mathbf{A}\left(\mathbf{M}_{\mathbf{r q}}\right)_{i} \mathbf{A}^{-1} \\
\left(\mathbf{M}_{\mathbf{r q}} \mathbf{M}_{\mathbf{r r}}\right)_{o} & =-\mathbf{A}\left(\mathbf{M}_{\mathbf{r r}}\right)_{i} \mathbf{A}^{t} \mathbf{L} \mathbf{A}\left(\mathbf{M}_{\mathbf{r r}}\right)_{i} \mathbf{A}^{t}+\mathbf{A}\left(\mathbf{M}_{\mathbf{r q}} \mathbf{M}_{\mathbf{r r}}\right)_{i} \mathbf{A}^{t} .
\end{aligned}
$$

From Eq. (40a) we see that

$$
\left(\operatorname{det} \mathbf{M}_{\mathbf{r r}}\right)_{o}^{1 / 2}=\left(\operatorname{det} \mathbf{M}_{\mathbf{r r}}\right)_{i}^{1 / 2} \operatorname{det} \mathbf{A} .
$$

As the matrix $\mathbf{A}\left(\mathbf{M}_{\mathbf{r r}}\right)_{i} \mathbf{A}^{t} \mathbf{L} \mathbf{A}\left(\mathbf{M}_{\mathbf{r r}}\right)_{i} \mathbf{A}^{t}$ in Eq. (40c) is symmetric, the asymmetry of $\left(\mathbf{M}_{\mathbf{r q}} \mathbf{M}_{\mathbf{r r}}\right)_{O}$ is equal to the asymmetry of $\mathbf{A}\left(\mathbf{M}_{\mathbf{r q}} \mathbf{M}_{\mathbf{r r}}\right)_{i} \mathbf{A}^{t}$, which in its turn is equal to the asymmetry of $\left(\mathbf{M}_{\mathbf{r q}} \mathbf{M}_{\mathbf{r r}}\right)_{i} \operatorname{det} \mathbf{A}$. Both the numerator and the denominator in the expression for $T$, see Eq. (35), scale with the same factor $\operatorname{det} \mathbf{A}$, and we thus conclude that the twist $T_{o}$ in the output plane equals the twist $T_{i}$ in the input plane.

Since $T$ is an invariant between conjugate planes, the twist of the beam with moment matrix $\mathbf{M}$ is identical to the twist of its generalized canonical form $\mathbf{M}^{\mathbf{0}}$ and its conjugate canonical form $\overline{\mathbf{M}}^{\mathrm{o}}$. We thus have found a direct way to express the latitude $2 \beta$ of the generalized canonical form on the Poincaré sphere (or its component $Q_{3}$ $=Q \sin 2 \beta=2 m_{x v}^{0}$ ) in terms of the entries of the moment matrix M. We only need to determine the canonical eigenvalues $\lambda_{x, y}$ via Eq. (11) and the twist $T$ via Eq. (35), and then use Eqs. (37) and (38) to get

$$
Q_{3}=\left(\lambda_{x}-\lambda_{y}\right) \sin 2 \beta=\frac{2 T \sqrt{\lambda_{x} \lambda_{y}}}{\sqrt{\left(\lambda_{x}+\lambda_{y}\right)^{2}-T^{2}}} .
$$

A plot of $Q_{3} /\left(\lambda_{x}-\lambda_{y}\right)$ versus $T /\left(\lambda_{x}-\lambda_{y}\right)$ for different values of the ratio $\left(\lambda_{x}-\lambda_{y}\right)^{2} / 4 \lambda_{x} \lambda_{y}$ has been depicted in Fig. 1.

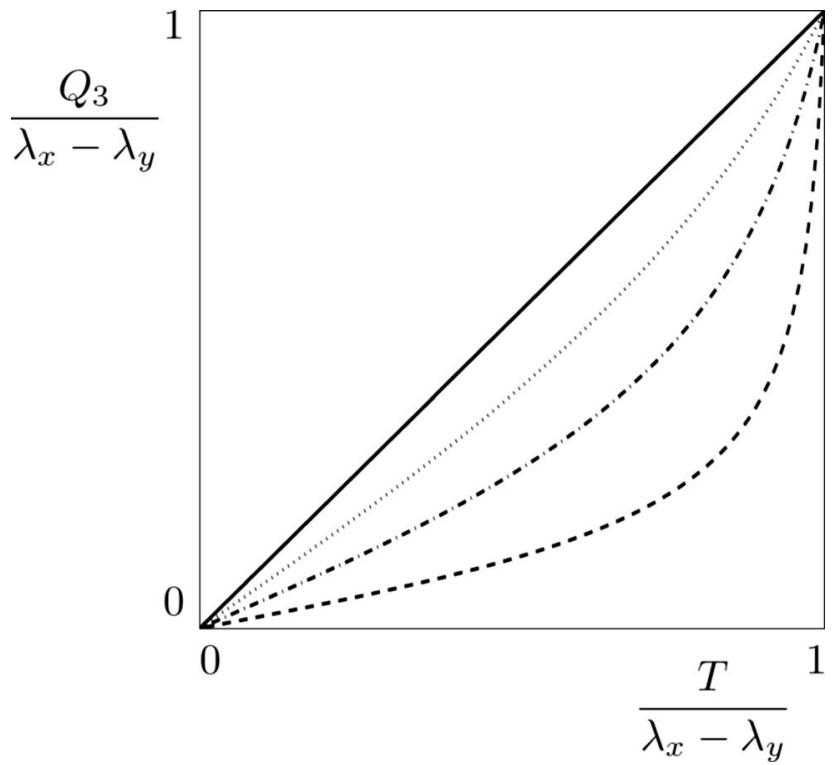

Fig. 1. Plot of $Q_{3} /\left(\lambda_{x}-\lambda_{y}\right)$ versus $T /\left(\lambda_{x}-\lambda_{y}\right)$ for different values of the ratio $r^{2}=\left(\lambda_{x}-\lambda_{y}\right)^{2} / 4 \lambda_{x} \lambda_{y}: r=5$ (dashed curve), $r=2$ (dasheddotted curve), $r=1$ (dotted curve), and $r \rightarrow 0$ (solid curve).

\section{B. Propagation of the Orbital Angular Momentum in an Isotropic System}

We recall that the orbital angular momentum $\Lambda$ is invariant in an isotropic first-order optical system [28-30], for which the ray transformation matrix can be expressed as

$$
\mathbf{T}=\left[\begin{array}{ll}
a \mathbf{U}_{r}(-\alpha) & b \mathbf{U}_{r}(-\alpha) \\
c \mathbf{U}_{r}(-\alpha) & d \mathbf{U}_{r}(-\alpha)
\end{array}\right] \quad \text { with } a d-b c=1 .
$$

Such an invariance does not generally hold for the twist, but it may hold if the signal satisfies additional conditions. In the case of a rotationally symmetric beam, for instance, for which [31]

$$
\mathbf{M}_{\mathbf{r r}}=m_{x x} \mathbf{I}, \quad \mathbf{M}_{\mathbf{q q}}=m_{u u} \mathbf{I}, \quad \mathbf{M}_{\mathbf{r q}}=\left[\begin{array}{cc}
m_{x u} & m_{x v} \\
-m_{x v} & m_{x u}
\end{array}\right],
$$

we easily verify that the twist $T$ equals the orbital angular momentum $\Lambda=2 m_{x v}$ and is thus invariant.

\section{GENERAL TWO-DIMENSIONAL CASE}

In the most general case, we can find the system that brings the moment matrix $\mathbf{M}$ into its canonical form $\Delta$, still without having to determine the symplectic matrix $\mathbf{T}^{\mathbf{o}}$ whose columns are the eigenvectors of $(\mathbf{M J})^{2}$, see Eq. (9). We start with Eq. (34). Using the canonical decomposition for the block-diagonal inner matrix, which is positive definite symmetric like $\mathbf{M}$ itself, this inner matrix can be written in the form

$$
\left[\begin{array}{cc}
\mathbf{M}_{\mathbf{r r}} & \mathbf{0} \\
\mathbf{0} & \mathbf{M}_{\mathbf{q q}}-\mathbf{M}_{\mathbf{r q}}^{t} \mathbf{M}_{\mathbf{r r}}^{-1} \mathbf{M}_{\mathbf{r q}}
\end{array}\right]=\mathbf{T}_{m}(\mathbf{S}) \mathbf{T}_{r}(-\alpha) \mathbf{G} \mathbf{T}_{r}(\alpha) \mathbf{T}_{m}(\mathbf{S})
$$

say, where the canonical form $\mathbf{G}$ contains the canonical eigenvalues $g_{x, y}$ of this inner matrix, and where the magnifier matrix $\mathbf{S}$ and the rotator angle $\alpha$ can be determined along the lines described in Appendix A. We can thus ex- 
press the moment matrix $\mathbf{M}$ as

$$
\begin{aligned}
\mathbf{M}= & \mathbf{T}_{l^{\prime}}\left(-\mathbf{M}_{\mathbf{r q}}^{t} \mathbf{M}_{\mathbf{r r}}^{-1}\right) \mathbf{T}_{m}(\mathbf{S}) \mathbf{T}_{r}(-\alpha) \mathbf{G} \mathbf{T}_{r}(\alpha) \\
& \times \mathbf{T}_{m}(\mathbf{S}) \mathbf{T}_{l^{\prime}}^{t}\left(-\mathbf{M}_{\mathbf{r q}}^{t} \mathbf{M}_{\mathbf{r r}}^{-1}\right)
\end{aligned}
$$

where

$$
\mathbf{T}_{l^{\prime}}\left(-\mathbf{M}_{\mathbf{r q}}^{t} \mathbf{M}_{\mathbf{r r}}^{-1}\right)=\left[\begin{array}{cc}
\mathbf{I} & \mathbf{0} \\
\mathbf{M}_{\mathbf{r q}}^{t} \mathbf{M}_{\mathbf{r r}}^{-1} & \mathbf{I}
\end{array}\right] .
$$

Note that the matrix $\mathbf{T}_{l^{\prime}}\left(-\mathbf{M}_{\mathbf{r q}}^{t} \mathbf{M}_{\mathbf{r r}}^{-1}\right)$ corresponds to the ray transformation matrix $\mathbf{T}_{l}$ of a lens, and that the eigenvalues $g_{x, y}$ are equal to the canonical eigenvalues $\lambda_{x, y}$ of $\mathbf{M}$ only if the matrix $\mathbf{M}_{\mathbf{r q}}^{t} \mathbf{M}_{\mathbf{r r}}^{-1}$ is symmetric.

We now separate the (possibly asymmetric) matrix $-\mathbf{M}_{\mathbf{r q}}^{t} \mathbf{M}_{\mathbf{r r}}^{-1}$ into its symmetric and antisymmetric parts,

$$
\begin{aligned}
-\mathbf{M}_{\mathbf{r q}}^{t} \mathbf{M}_{\mathbf{r r}}^{-1} & =\mathbf{L}_{s}+h \boldsymbol{\sigma} \text { with } \boldsymbol{\sigma}=\left[\begin{array}{cc}
0 & 1 \\
-1 & 0
\end{array}\right], \\
\mathbf{L}_{s} & =-\frac{1}{2}\left(\mathbf{M}_{\mathbf{r q}}^{t} \mathbf{M}_{\mathbf{r r}}^{-1}+\mathbf{M}_{\mathbf{r r}}^{-1} \mathbf{M}_{\mathbf{r q}}\right), \\
h \boldsymbol{\sigma} & =-\frac{1}{2}\left(\mathbf{M}_{\mathbf{r q}}^{t} \mathbf{M}_{\mathbf{r r}}^{-1}-\mathbf{M}_{\mathbf{r r}}^{-1} \mathbf{M}_{\mathbf{r q}}\right),
\end{aligned}
$$

leading to

$$
\begin{aligned}
\mathbf{M}= & \mathbf{T}_{l}\left(\mathbf{L}_{s}\right) \mathbf{T}_{l^{\prime}}(h \boldsymbol{\sigma}) \mathbf{T}_{m}(\mathbf{S}) \mathbf{T}_{r}(-\alpha) \\
& \times \mathbf{G} \mathbf{T}_{r}(\alpha) \mathbf{T}_{m}(\mathbf{S}) \mathbf{T}_{l^{\prime}}^{t}(h \boldsymbol{\sigma}) \mathbf{T}_{l}^{t}\left(\mathbf{L}_{s}\right) .
\end{aligned}
$$

Note that the matrix $\mathbf{T}_{l}\left(\mathbf{L}_{s}\right)$ corresponds to the ray transformation matrix of a lens, but that the matrix $\mathbf{T}_{l^{\prime}}(h \boldsymbol{\sigma})$ does not (unless $h$ vanishes). By shifting the matrices $\mathbf{T}_{l^{\prime}}(h \boldsymbol{\sigma})$ and $\mathbf{T}_{l^{\prime}}^{t}(h \boldsymbol{\sigma})$ toward $\mathbf{G}$, expressing the cascade $\mathbf{T}_{l^{\prime}}(h \boldsymbol{\sigma} \operatorname{det} \mathbf{S}) \mathbf{G T}_{l^{\prime}}^{t}(h \boldsymbol{\sigma} \operatorname{det} \mathbf{S})$ in its canonical form $\boldsymbol{\Delta}$ and rearranging the occurring ray transformation matrices, we get the final form

$$
\begin{aligned}
\mathbf{M}= & \mathbf{T}_{l}\left(\mathbf{L}_{s}+\mathbf{L}_{a}\right) \mathbf{T}_{m}(m \mathbf{S}) \mathbf{T}_{r}(-\alpha) \mathbf{T}_{g}(-\beta) \Delta \mathbf{T}_{g}(\beta) \\
& \times \mathbf{T}_{r}(\alpha) \mathbf{T}_{m}(m \mathbf{S}) \mathbf{T}_{l}^{t}\left(\mathbf{L}_{s}+\mathbf{L}_{a}\right),
\end{aligned}
$$

where

$$
\begin{aligned}
\mathbf{L}_{a} & =\ell \mathbf{S}^{-1}\left[\begin{array}{cc}
-\sin 2 \alpha & \cos 2 \alpha \\
\cos 2 \alpha & \sin 2 \alpha
\end{array}\right] \mathbf{S}^{-1}, \\
\text { with } \ell & =\frac{g_{x}-g_{y}}{g_{x}+g_{y}}(h \operatorname{det} \mathbf{S}) \\
& =\frac{-T}{2 \sqrt{\lambda_{x} \lambda_{y}}} \sqrt{\frac{\left(\lambda_{x}-\lambda_{y}\right)^{2}-T^{2}}{\left(\lambda_{x}+\lambda_{y}\right)^{2}-T^{2}}}, \\
\text { and } m^{-4} & =1+\frac{4 g_{x} g_{y}}{\left(g_{x}+g_{y}\right)^{2}}(h \operatorname{det} \mathbf{S})^{2} \\
& =1+\frac{T^{2}}{\sqrt{\left(\lambda_{x}+\lambda_{y}\right)^{2}-T^{2}}} .
\end{aligned}
$$

For the details how to convert Eq. (47) into Eq. (48), we refer to Appendix B.

The lens $\mathcal{L}\left(\mathbf{L}_{a}\right)$ that appears in addition to the lens $\mathcal{L}\left(\mathbf{L}_{s}\right)$ is proportional to the asymmetry $-h$ of the matrix
$\mathbf{M}_{\mathbf{r q}}^{t} \mathbf{M}_{\mathbf{r r}}^{-1}$. When $\mathbf{M}_{\mathbf{r q}}^{t} \mathbf{M}_{\mathbf{r r}}^{-1}$ is symmetric, this additional lens vanishes, and so does the gyrator; the generalized canonical form $\mathbf{M}^{\mathrm{o}}=\mathbf{T}_{r}(-\alpha) \Delta \mathbf{T}_{r}(\alpha)$ is then located on the equator of the Poincaré sphere. Note that the additional lens also vanishes when $g_{x}=g_{y}=g$, in which case $|T|=\lambda_{x}-\lambda_{y}=2 g|\widetilde{h}|$ and $|\beta|=\pi / 4$, cf. Eqs. (B6a), (B6c), and (B7) in Appendix B; the generalized canonical form $\mathbf{M}^{\mathrm{o}}=\mathbf{T}_{g}( \pm \pi / 4) \boldsymbol{\Delta} \mathbf{T}_{g}(\mp \pi / 4)$, where the sign depends on the sign of $h$, is then located at one of the poles of the Poincaré sphere.

\section{CONCLUSION}

In this paper we have generalized the way in which Gaussian-type modes are represented on the angular Poincaré sphere, to general two-dimensional signals. It was shown that, with the help of a lens and a magnifier, any two-dimensional beam can be transformed into a beam for which the matrix of second-order moments has the generalized canonical form $\mathbf{M}^{0}$. By ordering the entries of $\mathbf{M}^{\mathrm{o}}$ in such a way that the three components of the beam's orbital angular momentum arise, and based on the way in which a rotator and a gyrator act on a beam in its canonical form $\Delta$, we were able to locate the beam on a certain point on the Poincaré sphere. We have shown an easy way how the entries of the diagonal matrix $\Delta$, i.e., the canonical eigenvalues $\lambda_{x}$ and $\lambda_{y}$, can be derived directly from the second-order moments; see also [10].

We have then shown that the intrinsically isotropic case-i.e., the case for which the canonical form takes the form of a scalar matrix, $\Delta=\lambda \mathbf{I}$, and the Poincaré sphere reduces to a point-corresponds to the case that $\lambda^{-1} \mathbf{M}$ is a symplectic matrix. The moment matrix has then only seven degrees of freedom, in accordance with (i) the three degrees of freedom of the lens, (ii) the three degrees of freedom of the magnifier, and (iii) the canonical eigenvalue $\lambda_{x}=\lambda_{y}=\lambda$. The lens and the magnifier follow easily from the moment matrix $\mathbf{M}$.

Subsequently, we have studied the case for which the generalized canonical form $\mathbf{M}^{0}$ is located on the equator of the Poincaré sphere, and we have shown that this case corresponds to a moment matrix $\mathbf{M}$ with zero-twist. The moment matrix then satisfies the symmetry condition $\mathbf{M}_{\mathbf{r q}} \mathbf{M}_{\mathbf{r r}}=\mathbf{M}_{\mathbf{r r}} \mathbf{M}_{\mathbf{r q}}^{t}$ and has nine degrees of freedom, in accordance with (i) the three degrees of freedom of the lens, (ii) the three degrees of freedom of the magnifier, (iii) the two canonical eigenvalues, and (iv) an additional rotation along the equator. We have shown a direct way to determine the lens, the magnifier, and the rotation angle from the second-order moments.

In the most general case, with the moment matrix having the full ten degrees of freedom, the generalized canonical form may be located off the equator, i.e., its latitude may be nonzero. The general case corresponds to a moment matrix that has twist, and we have related the degree of twist to the latitude of the generalized canonical form. In particular, we were able to express the latitude directly in terms of the canonical eigenvalues $\lambda_{x, y}$ and the twist $T$. We have also shown that the twist parameter $T$ of a beam, whose value is bounded by $\pm\left(\lambda_{x}-\lambda_{y}\right)$, is invariant when this beam propagates through a first-order optical system between conjugate planes. Due to this invariance 
and its easy measurability, the twist parameter is a good candidate to describe the vortex part of the orbital angular momentum.

Finally, we have shown a direct way to find an optical system (in general consisting of a lens, a magnifier, a rotator, and a gyrator) that transforms a beam with an arbitrary moment matrix into its canonical form. In the resulting cascade, the lens cancels the quadratic phase front of the initial beam, the subsequent magnifier matches the space moments and the spatial-frequency moments of the beam, and the rotator aligns the beam with its principal axes.

\section{APPENDIX A: THE CASE $\beta=0$}

When $\mathbf{M}_{\mathbf{r q}} \mathbf{M}_{\mathbf{r r}}=\mathbf{M}_{\mathbf{r r}} \mathbf{M}_{\mathbf{r q}}^{t}$, which results in $\beta=0$, we can determine the lens $\mathcal{L}(\mathbf{L})$, the magnifier $\mathcal{M}(\mathbf{S})$, and the rotator $\mathcal{R}(-\alpha)$ directly from the entries of the moment matrix. The matrix $\mathbf{L}$ follows immediately as $\mathbf{L}=-\mathbf{M}_{\mathbf{r r}}^{-1} \mathbf{M}_{\mathbf{r q}}$, cf. Eqs. (27a) and (27b). To determine the remaining variables $\mathbf{S}$ and $\alpha$, let us consider the reduced matrix $\mathbf{M}^{\mathbf{L}}$, cf. Eq. (27a):

$$
\mathbf{M}^{\mathbf{L}}=\left[\begin{array}{cc}
\mathbf{M}_{\mathbf{r r}} & \mathbf{M}_{\mathbf{r q}}^{\mathbf{L}} \\
\left(\mathbf{M}_{\mathbf{r q}}^{\mathbf{L}}\right)^{t} & \mathbf{M}_{\mathbf{q q}}^{\mathbf{L}}
\end{array}\right]=\left[\begin{array}{cc}
\mathbf{I} & \mathbf{0} \\
\mathbf{L} & \mathbf{I}
\end{array}\right] \mathbf{M}\left[\begin{array}{cc}
\mathbf{I} & \mathbf{L} \\
\mathbf{0} & \mathbf{I}
\end{array}\right] .
$$

We recall that $\operatorname{det} \overline{\mathbf{M}}_{\mathbf{r r}}^{0}(0)=\lambda_{x} \lambda_{y}=\sqrt{\operatorname{det} \mathbf{M}}$, see Eqs. (26) and (10a).

The positive-definite symmetric matrix $\mathbf{S}$ can be expressed in its eigenvalues and eigenvectors as

$$
\mathbf{S}=\left[\begin{array}{ll}
s_{x x} & s_{x y} \\
s_{x y} & s_{y y}
\end{array}\right]=\left[\begin{array}{cc}
\cos \theta & -\sin \theta \\
\sin \theta & \cos \theta
\end{array}\right]\left[\begin{array}{cc}
s_{x} & 0 \\
0 & s_{y}
\end{array}\right]\left[\begin{array}{cc}
\cos \theta & \sin \theta \\
-\sin \theta & \cos \theta
\end{array}\right],
$$

where $s_{x, y} \geqslant 0$. As a simple example, we mention the separable case, $m_{x y}=m_{u v}^{\mathbf{L}}=0$, for which we have $\left(\bar{m}_{x x}^{0}\right)^{2}$ $=m_{x x} m_{u u}^{\mathbf{L}}$ and $\left(\bar{m}_{y y}^{0}\right)^{2}=m_{y y} m_{v v}^{\mathbf{L}}$, and for which we easily verify $s_{x}^{4}=m_{x x} / m_{u u}^{\mathbf{L}}, s_{y}^{4}=m_{y y} / m_{v v}^{\mathbf{L}}$, and $\theta=\alpha=0$.

The non-separable case is not so easy, but we do have the relation $\mathbf{S}^{-2} \mathbf{M}_{\mathbf{r r}}=\mathbf{M}_{\mathbf{q q}}^{\mathbf{L}} \mathbf{S}^{2}$, see Eq. (29), from which the symmetric matrix $\mathbf{S}^{2}$ can be determined. If we define $\mathbf{P}$ $=\mathbf{S}^{2}$,

$$
\mathbf{P}=\left[\begin{array}{ll}
p_{x x} & p_{x y} \\
p_{x y} & p_{y y}
\end{array}\right]=\left[\begin{array}{cc}
\cos \theta & -\sin \theta \\
\sin \theta & \cos \theta
\end{array}\right]\left[\begin{array}{cc}
s_{x}^{2} & 0 \\
0 & s_{y}^{2}
\end{array}\right]\left[\begin{array}{cc}
\cos \theta & \sin \theta \\
-\sin \theta & \cos \theta
\end{array}\right],
$$

we can write the relation $\mathbf{S}^{-2} \mathbf{M}_{\mathbf{r r}}=\mathbf{M}_{\mathbf{q q}}^{\mathbf{L}} \mathbf{S}^{2}$ in detail as

$$
\begin{gathered}
{\left[\begin{array}{cc}
p_{y y} & -p_{x y} \\
-p_{x y} & p_{x x}
\end{array}\right]\left[\begin{array}{ll}
m_{x x} & m_{x y} \\
m_{x y} & m_{y y}
\end{array}\right]} \\
=(\operatorname{det} \mathbf{S})^{2}\left[\begin{array}{ll}
m_{u u}^{\mathbf{L}} & m_{u v}^{\mathbf{L}} \\
m_{u v}^{\mathbf{L}} & m_{v v}^{\mathbf{L}}
\end{array}\right]\left[\begin{array}{ll}
p_{x x} & p_{x y} \\
p_{x y} & p_{y y}
\end{array}\right] \\
=:\left[\begin{array}{ll}
m_{u u}^{\prime} & m_{u v}^{\prime} \\
m_{u v}^{\prime} & m_{v v}^{\prime}
\end{array}\right]\left[\begin{array}{ll}
p_{x x} & p_{x y} \\
p_{x y} & p_{y y}
\end{array}\right],
\end{gathered}
$$

with $(\operatorname{det} \mathbf{S})^{2}=\operatorname{det} \mathbf{M}_{\mathbf{r r}} / \operatorname{det} \overline{\mathbf{M}}_{\mathbf{r r}}^{0}(0)$, cf. Eq. (30), and we get the relations

$$
\left[\begin{array}{ccc}
m_{u u}^{\prime} & m_{x y}+m_{u v}^{\prime} & -m_{x x} \\
0 & m_{y y}+m_{u u}^{\prime} & -m_{x y}+m_{u v}^{\prime} \\
-m_{x y}+m_{u v}^{\prime} & m_{x x}+m_{v v}^{\prime} & 0 \\
-m_{y y} & m_{x y}+m_{u v}^{\prime} & m_{v v}^{\prime}
\end{array}\right]\left[\begin{array}{c}
p_{x x} \\
p_{x y} \\
p_{y y}
\end{array}\right]=\mathbf{0}
$$

note that $m_{x x} m_{y y}-m_{x y}^{2}=m_{u u}^{\prime} m_{v v}^{\prime}-\left(m_{u v}^{\prime}\right)^{2}$. The $4 \times 3$ matrix in the latter expression has row dependence, $\left(m_{x y}-m_{u v}^{\prime}\right)$ $\times($ row 1 -row 4$)-\left(m_{x x}+m_{v v}^{\prime}\right)$ row $2+\left(m_{y y}+m_{u u}^{\prime}\right)$ row $3=0$, and we can reduce it to a $3 \times 3$ matrix, for instance, by deleting row 4 ,

$$
\left[\begin{array}{ccc}
m_{u u}^{\prime} & m_{x y}+m_{u v}^{\prime} & -m_{x x} \\
0 & m_{y y}+m_{u u}^{\prime} & -m_{x y}+m_{u v}^{\prime} \\
-m_{x y}+m_{u v}^{\prime} & m_{x x}+m_{v v}^{\prime} & 0
\end{array}\right]\left[\begin{array}{c}
p_{x x} \\
p_{x y} \\
p_{y y}
\end{array}\right]=\mathbf{0} .
$$

The determinant of this matrix vanishes, so that we have a vanishing eigenvalue.

If $m_{x y} \neq m_{u v}^{\prime}$, the entries of the eigenvector that is associated with the vanishing eigenvalue follow most easily from rows 2 and 3 ,

$$
\begin{aligned}
& p_{x x} / p_{x y}=\left(m_{x x}+m_{v v}^{\prime}\right) /\left(m_{x y}-m_{u v}^{\prime}\right), \\
& p_{y y} / p_{x y}=\left(m_{y y}+m_{u u}^{\prime}\right) /\left(m_{x y}-m_{u v}^{\prime}\right),
\end{aligned}
$$

and shall be normalized according to $p_{x x} p_{y y}-p_{x y}^{2}$ $=\operatorname{det} \mathbf{M}_{\mathbf{r r}} / \operatorname{det} \overline{\mathbf{M}}_{\mathbf{r r}}^{\mathrm{o}}(0)$. Hence

$$
p_{x y}^{2}\left[\frac{\left(m_{x x}+m_{v v}^{\prime}\right)\left(m_{y y}+m_{u u}^{\prime}\right)}{\left(m_{x y}-m_{u v}^{\prime}\right)^{2}}-1\right]=\frac{\operatorname{det} \mathbf{M}_{\mathbf{r r}}}{\sqrt{\operatorname{det} \mathbf{M}}}
$$

and the sign of $p_{x y}$ should be chosen equal to the sign of $m_{x y}-m_{u v}^{\prime}$. We thus get explicit values for $p_{x x}, p_{x y}$, and $p_{y y}$. Once $\mathbf{P}$ has been found, we easily conclude from Eq. (A3)

$$
\begin{aligned}
s_{x, y}^{2} & =\frac{1}{2}\left(p_{x x}+p_{y y}\right) \pm \frac{1}{2} \sqrt{\left(p_{x x}-p_{y y}\right)^{2}+p_{x y}^{2}}, \\
\tan \theta & =\left(s_{x}^{2}-p_{x x}\right) / p_{x y}=p_{x y} /\left(s_{x}^{2}-p_{y y}\right)=p_{x y} /\left(p_{x x}-s_{y}^{2}\right) \\
& =\left(p_{y y}-s_{y}^{2}\right) / p_{x y},
\end{aligned}
$$

and the matrix $\mathbf{S}$ follows from Eq. (A2).

If $m_{x y}=m_{u v}^{\prime}$, things are even easier: we get $p_{x y}=0$, the matrix $\mathbf{P}$ becomes a diagonal matrix, $\theta=0$, and from $\mathbf{M}_{\mathbf{r r}}$ $=\mathbf{P M}_{\mathbf{q q}}^{\mathbf{L}} \mathbf{P}$ we get immediately

$$
p_{x x}^{2}=m_{x x} / m_{u u}^{\mathbf{L}}=s_{x}^{4} \quad \text { and } \quad p_{y y}^{2}=m_{y y} / m_{v v}^{\mathbf{L}}=s_{y}^{4} .
$$

We finally derive $\alpha$ from Eq. (29).

\section{APPENDIX B: FROM EQ. (47) TO EQ. (48)}

Let us start with Eq. (47), which we repeat here for convenience:

$$
\begin{aligned}
\mathbf{M}= & \mathbf{T}_{l}\left(\mathbf{L}_{s}\right) \mathbf{T}_{l^{\prime}}(h \boldsymbol{\sigma}) \mathbf{T}_{m}(\mathbf{S}) \mathbf{T}_{r}(-\alpha) \\
& \times \mathbf{G} \mathbf{T}_{r}(\alpha) \mathbf{T}_{m}(\mathbf{S}) \mathbf{T}_{l^{\prime}}^{t}(h \boldsymbol{\sigma}) \mathbf{T}_{l}^{t}\left(\mathbf{L}_{s}\right)
\end{aligned}
$$

Using the identity 
$\mathbf{T}_{l^{\prime}}(h \boldsymbol{\sigma}) \mathbf{T}_{m}(\mathbf{S}) \mathbf{T}_{r}(-\alpha)=\mathbf{T}_{m}(\mathbf{S}) \mathbf{T}_{r}(-\alpha) \mathbf{T}_{l^{\prime}}(h \boldsymbol{\sigma} \operatorname{det} \mathbf{S})$

we can change the order of the matrices in the cascade (B1) and write

$$
\begin{aligned}
\mathbf{M}= & \mathbf{T}_{l}\left(\mathbf{L}_{s}\right) \mathbf{T}_{m}(\mathbf{S}) \mathbf{T}_{r}(-\alpha) \mathbf{T}_{l^{\prime}}(\tilde{h} \boldsymbol{\sigma}) \\
& \times \mathbf{G} \mathbf{T}_{l^{\prime}}^{t}(\tilde{h} \boldsymbol{\sigma}) \mathbf{T}_{r}(\alpha) \mathbf{T}_{m}(\mathbf{S}) \mathbf{T}_{l}^{t}\left(\mathbf{L}_{s}\right),
\end{aligned}
$$

where we have introduced $\widetilde{h}=h \operatorname{det} \mathbf{S}$.

The cascade of the inner three matrices, $\mathbf{T}_{l^{\prime}}(\tilde{h} \boldsymbol{\sigma}) \mathbf{G} \mathbf{T}_{l^{\prime}}^{t}(\tilde{h} \boldsymbol{\sigma})$, takes the form

$$
\left[\begin{array}{cccc}
g_{x} & 0 & 0 & -g_{x} \tilde{h} \\
0 & g_{y} & g_{y} \tilde{h} & 0 \\
0 & g_{y} \widetilde{h} & g_{x}+g_{y} \widetilde{h}^{2} & 0 \\
-g_{x} \widetilde{h} & 0 & 0 & g_{y}+g_{x} \widetilde{h}^{2}
\end{array}\right] .
$$

Using the canonical decomposition again, the latter matrix can be written in the form

$$
\mathbf{T}_{l}\left(\mathbf{L}^{\prime}\right) \mathbf{T}_{m}\left(\mathbf{S}^{\prime}\right) \mathbf{T}_{g}(-\beta) \Delta \mathbf{T}_{g}(\beta) \mathbf{T}_{m}\left(\mathbf{S}^{\prime}\right) \mathbf{T}_{l}^{t}\left(\mathbf{L}^{\prime}\right),
$$

where the lens matrix $\mathbf{L}^{\prime}$ and the magnifier matrix $\mathbf{S}^{\prime}$ follow from $g_{x, y}$ and $\widetilde{h}$ via

$$
\begin{aligned}
& \mathbf{L}^{\prime}=\left[\begin{array}{ll}
0 & \ell \\
\ell & 0
\end{array}\right] \quad \text { with } \quad \ell=\frac{g_{x}-g_{y}}{g_{x}+g_{y}} \widetilde{h}, \\
& \mathbf{S}^{\prime}=m \mathbf{I} \quad \text { with } \quad m^{-4}=1+\frac{4 g_{x} g_{y}}{\left(g_{x}+g_{y}\right)^{2}} \widetilde{h}^{2},
\end{aligned}
$$

see Eqs. (49b) and (49c). The moment matrix M thus takes the form

$$
\begin{aligned}
\mathbf{M}= & \mathbf{T}_{l}\left(\mathbf{L}_{s}\right) \mathbf{T}_{m}(\mathbf{S}) \mathbf{T}_{r}(-\alpha) \mathbf{T}_{l}\left(\mathbf{L}^{\prime}\right) \mathbf{T}_{m}(m \mathbf{I}) \mathbf{T}_{g}(-\beta) \Delta \\
& \times \mathbf{T}_{g}(\beta) \mathbf{T}_{m}(m \mathbf{I}) \mathbf{T}_{l}^{t}\left(\mathbf{L}^{\prime}\right) \mathbf{T}_{r}(\alpha) \mathbf{T}_{m}(\mathbf{S}) \mathbf{T}_{l}^{t}\left(\mathbf{L}_{s}\right),
\end{aligned}
$$

where all matrices $\mathbf{T}$ now correspond to real optical systems, as is required for a canonical decomposition.

Using the identity

$$
\begin{aligned}
& \mathbf{T}_{m}(\mathbf{S}) \mathbf{T}_{r}(-\alpha) \mathbf{T}_{l}\left(\mathbf{L}^{\prime}\right) \mathbf{T}_{m}(m \mathbf{I}) \\
& \quad=\mathbf{T}_{l}\left[\mathbf{S}^{-1} \mathbf{U}_{r}(-\alpha) \mathbf{L}^{\prime} \mathbf{U}_{r}(\alpha) \mathbf{S}^{-1}\right] \mathbf{T}_{m}(m \mathbf{S}) \mathbf{T}_{r}(-\alpha),
\end{aligned}
$$

we can change the order of the matrices in the cascade (B4) and write $\mathbf{M}$ in its final form,

$$
\begin{aligned}
\mathbf{M}= & \mathbf{T}_{l}\left(\mathbf{L}_{s}+\mathbf{L}_{a}\right) \mathbf{T}_{m}(m \mathbf{S}) \mathbf{T}_{r}(-\alpha) \mathbf{T}_{g}(-\beta) \Delta \mathbf{T}_{g}(\beta) \\
& \times \mathbf{T}_{r}(\alpha) \mathbf{T}_{m}(m \mathbf{S}) \mathbf{T}_{l}^{t}\left(\mathbf{L}_{s}+\mathbf{L}_{a}\right),
\end{aligned}
$$

see Eq. (48), where we have introduced the lens matrix

$$
\mathbf{L}_{a}=\mathbf{S}^{-1} \mathbf{U}_{r}(-\alpha) \mathbf{L}^{\prime} \mathbf{U}_{r}(\alpha) \mathbf{S}^{-1},
$$

see Eq. (49a).

To express $\ell$ and $m$ in terms of the canonical eigenvalues $\lambda_{x, y}$ and the twist $T$, we apply Eqs. (10a), (10c), and (35) to the matrix (B3). We thus easily verify the relations between $\lambda_{x, y}$ and $T$ on the one hand and $g_{x, y}$ and $\tilde{h}$ on the other as

$$
\left(\lambda_{x} \pm \lambda_{y}\right)^{2}=\left(g_{x} \pm g_{y}\right)^{2}+4 g_{x} g_{y} \widetilde{h}^{2},
$$

$$
\lambda_{x} \lambda_{y}=g_{x} g_{y}
$$

$$
T=-2 \widetilde{h} \sqrt{g_{x} g_{y}},
$$

so that

$$
\ell=\frac{-T}{2 \sqrt{\lambda_{x} \lambda_{y}}} \sqrt{\frac{\left(\lambda_{x}-\lambda_{y}\right)^{2}-T^{2}}{\left(\lambda_{x}+\lambda_{y}\right)^{2}-T^{2}}}
$$

$$
\text { and } m^{-4}=1+\frac{T^{2}}{\sqrt{\left(\lambda_{x}+\lambda_{y}\right)^{2}-T^{2}}},
$$

see Eqs. (49b) and (49c). For completeness, we mention the expression for the latitude $2 \beta$ in terms of $g_{x, y}$ and $\widetilde{h}$ :

$$
\sin 2 \beta=\frac{-4 g_{x} g_{y} \tilde{h}}{\left(g_{x}+g_{y}\right) \sqrt{\left(g_{x}-g_{y}\right)^{2}+4 g_{x} g_{y} \widetilde{h}^{2}}} .
$$

Note that for $g_{x}=g_{y}$, we have $\sin 2 \beta=-\operatorname{sgn}(\tilde{h})$.

\section{ACKNOWLEDGMENTS}

The financial support of the Spanish Ministry of Science and Innovation under project TEC2008-04105 and the Santander-Complutense project PR-34/07-15914 is acknowledged.

\section{REFERENCES}

1. M. Born and E. Wolf, Principles of Optics (Pergamon, 1980).

2. M. J. Padgett and J. Courtial, "Poincaré-sphere equivalent for light beams containing orbital angular momentum," Opt. Lett. 24, 430-432 (1999).

3. G. S. Agarwal, "SU(2) structure of the Poincaré sphere for light beams with orbital angular momentum," J. Opt. Soc. Am. A 16, 2914-2916 (1999)

4. G. F. Calvo, "Wigner representation and geometric transformations of optical orbital angular momentum spatial modes," Opt. Lett. 30, 1207-1209 (2005).

5. T. Alieva and M. J. Bastiaans, "Orthonormal mode sets for the two-dimensional fractional Fourier transformation," Opt. Lett. 32, 1226-1228 (2007).

6. T. Alieva and M. J. Bastiaans, "Phase-space rotations and orbital Stokes parameters," Opt. Lett. 34, 410-412 (2009).

7. M. J. Bastiaans, "Application of the Wigner distribution function to partially coherent light," J. Opt. Soc. Am. A 3, 1227-1238 (1986).

8. M. J. Bastiaans, "Second-order moments of the Wigner distribution function in first-order optical systems," Optik $\mathbf{8 8}$ 163-168 (1991).

9. J. Serna, R. Martínez-Herrero, and P. M. Mejías, "Parametric characterization of general partially coherent beams propagating through $A B C D$ optical systems," J. Opt. Soc. Am. A 8, 1094-1098 (1991).

10. K. Sundar, N. Mukunda, and R. Simon, "Coherent-mode decomposition of general anisotropic Gaussian Schell-model beams," J. Opt. Soc. Am. A 12, 560-569 (1995).

11. J. Williamson, "On the algebraic problem concerning the normal forms of linear dynamical systems," Am. J. Math. 58, 141-163 (1936).

12. R. K. Luneburg, Mathematical Theory of Optics (Univ. of California Press, 1966)

13. S. Helgason, Differential Geometry, Lie Groups and Symmetric Species (Academic, 1978), Chap. VI.

14. K. B. Wolf, Geometric Optics on Phase Space (Springer, 2004).

15. R. Simon and N. Mukunda, "Iwasawa decomposition in 
first-order optics: universal treatment of shape-invariant propagation for coherent and partially coherent beams," J. Opt. Soc. Am. A 15, 2146-2155 (1998).

16. R. Simon and K. B. Wolf, "Structure of the set of paraxial optical systems," J. Opt. Soc. Am. A 17, 342-355 (2000).

17. R. Simon and N. Mukunda, "Twisted Gaussian Schellmodel beams," J. Opt. Soc. Am. A 10, 95-109 (1993).

18. R. Simon, K. Sundar, and N. Mukunda, "Twisted Gaussian Schell-model beams. I. Symmetry structure and normalmode spectrum," J. Opt. Soc. Am. A 10, 2008-2016 (1993).

19. K. Sundar, R. Simon, and N. Mukunda, "Twisted Gaussian Schell-model beams. II. Spectrum analysis and propagation characteristics,” J. Opt. Soc. Am. A 10, 2017-2023 (1993).

20. A. T. Friberg, B. Tervonen, and J. Turunen, "Interpretation and experimental demonstration of twisted Gaussian Schell-model beams," J. Opt. Soc. Am. A 11, 1818-1826 (1994).

21. D. Ambrosini, V. Bagini, F. Gori, and M. Santarsiero, "Twisted Gaussian Schell-model beams: a superposition model," J. Mod. Opt. 41, 1391-1399 (1994).

22. R. Simon, A. T. Friberg, and E. Wolf, "Transfer of radiance by twisted Gaussian Schell-model beams in paraxial systems," J. Eur. Opt. Soc. A: Pure Appl. Opt. 5, 331-343 (1996).

23. R. Simon and N. Mukunda, "Twist phase in Gaussian-beam optics," J. Opt. Soc. Am. A 15, 2373-2382 (1998).
24. M. J. Bastiaans, "Wigner distribution function applied to twisted Gaussian light propagating in first-order optical systems," J. Opt. Soc. Am. A 17, 2475-2480 (2000).

25. M. S. Soskin, V. N. Gorshkov, M. V. Vasnetsov, J. T. Malos, and N. R. Heckenberg, "Topological charge and angular momentum of light beams carrying vortices," Phys. Rev. A 56, 163-165 (1997).

26. M. S. Soskin, M. V. Vasnetsov, "Singular optics," Prog. Opt. 42, 219-276 (2001).

27. J. Serna and J. M. Movilla, "Orbital angular momentum of partially coherent beams," Opt. Lett. 26, 405-407 (2001).

28. A. Ya. Bekshaev, M. V. Vasnetsov, V. G. Denisenko, and M. S. Soskin, "Transformation of the orbital angular momentum of a beam with optical vortex in a astigmatic optical system," JETP Lett. 75, 127-130 (2002).

29. A. Ya. Bekshaev, M. S. Soskin, and M. V. Vasnetsov, "Optical vortex symmetry breakdown and decomposition of the orbital angular momentum of the light beams," J. Opt. Soc. Am. A 20, 1635-1643 (2003).

30. T. Alieva and M. J. Bastiaans, "Evolution of the vortex and the asymmetrical parts of orbital angular momentum in separable first-order optical systems," Opt. Lett. 29, 15871589 (2004).

31. M. J. Bastiaans and T. Alieva, "Moments of the Wigner distribution of rotationally symmetric partially coherent light," Opt. Lett. 28, 2443-2445 (2003). 